\title{
Associação entre o estado nutricional e a prevalência de doenças crônicas não transmissíveis em idosos residentes no município de Roca Sales-RS
}

\author{
Bethania Neumann*, Simara Rufatto Conde*", \\ Joana Raquel Nunes Lemos"***, Thaís Rodrigues Moreira****
}

\section{Resumo}

O estudo objetiva avaliar o estado nutricional e associá-lo à prevalência de doenças crônicas não transmissíveis (DCNT) em idosos. Trata-se de um estudo transversal constituído por 112 indivíduos, com idade superior a 60 anos, de ambos os gêneros, participantes de grupos da terceira idade do município de Roca Sales-RS. Para avaliação do estado nutricional, foram aferidas circunferência da cintura (CC), peso e estatura. Após, foi calculado o índice de massa corporal (IMC). Aplicou-se um questionário para obtenção de informações sobre o estilo de vida e o diagnóstico de DCNT foi autorreferido pelos participantes. Observou-se que $85,7 \%(n=96)$ dos participantes eram do gênero feminino e $75,0 \% \quad(n=84)$ casados. Conforme o IMC, constatou-se que $57,1 \% \quad(n=64)$ apresentavam excesso de peso, evidenciando-se a maior média de IMC $(p=0,011)$ entre os homens. Na avaliação da CC, os homens também apresentaram maior média, quando comparados às mulheres $(p<0,001)$. A maioria dos participantes $(82,1 \%, \mathrm{n}=92)$ apresentava risco muito aumentado para complicações metabólicas. As DCNT diagnosticadas foram: hipertensão arterial sistêmica, dislipidemias, osteoporose e diabetes mellitus. Dos idosos avaliados, 83,0\% ( $\mathrm{n}=92)$ apresentavam pelo menos uma patologia e três foi o número médio de patologias. A partir dos resultados obtidos, verificou-se excesso de peso, risco muito aumentado para complicações metabólicas e diagnóstico de uma patologia na maioria dos indivíduos avaliados.

Palavras-chave: Estado nutricional. Doença crônica. Saúde do idoso.

* Nutricionista pelo Centro Universitário Univates. E-mail: bethania@universo.univates.br

** Nutricionista pela Universidade do Vale do Rio dos Sinos, Especialista em Educação em Saúde pelo Centro Universitário Univates, Mestre em Ciências Biológicas pela Universidade Federal do Rio Grande do Sul e Docente do curso de Nutrição do Centro Universitário Univates. E-mail: simararufatto@terra.com.br

**** Nutricionista pela Universidade do Vale do Rio dos Sinos, Especialista em Nutrição Clínica pela Universidade do Vale do Rio dos Sinos, Mestre em Ciências Médicas pela Universidade Federal do Rio Grande do Sul e Docente do curso de Nutrição do Centro Universitário Univates. E-mail: joanarnlemos@terra.com.br

**** Docente do curso de Nutrição do Centro Universitário Univates, Mestre em Ciências Médicas pela Universidade Federal do Rio Grande do Sul, Especialista em Nutrição Clínica pela Universidade Gama Filho e Pesquisadora do Serviço de Nefrologia do Hospital de Clínicas de Porto Alegre. E-mail: th_rodrigues@ ibest.com.br

$\rightarrow$ http://dx.doi.org/10.5335/rbceh.2012.4058

Recebido em: 22/06/2014. Aprovado em: 11/09/2014 


\section{Introdução}

O envelhecimento da população é considerado um fenômeno global resultante da redução da fertilidade e da mortalidade, do manejo de doenças transmissíveis, do avanço científico e do crescimento da tecnologia na área da saúde (FLORIANO et al., 2012). Conforme Cavalcanti et al. (2009), são considerados idosos, nos países em desenvolvimento, os indivíduos com faixa etária igual ou superior a 60 anos de idade, enquanto que, nos países desenvolvidos, a faixa é de 65 anos.

De acordo com o censo demográfico de 2010, o número de idosos no país foi de 20.590.599. As projeções para 2025 indicam que a população maior de 60 anos será superior a 30 milhões, com isso, o Brasil será o sexto país do mundo com maior número de idosos (FIGUEIREDO et al., 2012). O aumento da expectativa de vida leva a um maior número de idosos, e a um consequente aumento da prevalência de doenças crônicas entre essa população (LIMA et al., 2009). Aproximadamente $80 \%$ das pessoas acima de 65 anos apresentam ao menos uma patologia crônica e $10 \%$ desses idosos têm, no mínimo, cinco dessas patologias (PEDRAZZI; RODRIGUES; SCHIAVETO, 2007).

Apesar de o processo de envelhecimento não estar, necessariamente, relacionado a doenças e incapacidades, as doenças crônicas não transmissíveis (DCNT) são frequentemente encontradas entre os idosos (ALVES et al., 2007), tais como: doenças coronarianas, hipertensão arterial (HAS), diabetes mellitus (DM) tipo 2, acidentes vasculares encefálicos, doenças pulmonares crônicas e doenças osteoarticulares (OLIVEIRA et al., 2007).

Como afirma Veras (2011), muitas condições crônicas estão ligadas à sociedade em envelhecimento, mas também às escolhas de estilo de vida, como tabagismo, etilismo, comportamento sexual de risco, hábitos alimentares inadequados e inatividade física, além da predisposição genética. Nesse contexto, o estado nutricional interfere no envelhecimento e no desenvolvimento ou na prevenção de DCNT (NASCIMENTO et al., 2011). Sabe-se que indivíduos com excesso de peso têm risco de morbimortalidade por patologias cardiovasculares e $\mathrm{DM}$, assim como os indivíduos com baixo peso têm por patologias pulmonares e gástricas (WORLD HEALTH ORGANIZATION, 1995).

Com o aumento de pessoas com idade acima dos 60 anos, torna-se necessário o desenvolvimento de estudos que investiguem o perfil nutricional e a saúde, para que as propostas de educação continuada tenham adesão e impacto na qualidade de vida dos idosos (BUENO et al., 2008). Diante disso, o objetivo do presente estudo foi avaliar o estado nutricional e associá-lo à prevalência de doenças crônicas não transmissíveis em idosos do sul do país.

\section{Metodologia}

Trata-se de um estudo observacional transversal, realizado entre os meses de abril e maio de 2013. A amostra foi por conveniência e constituída por 112 indivíduos, com idade superior a 60 anos, de ambos os gêneros, participantes de grupos da terceira idade situados 
na área urbana do município de Roca Sales-RS. O estudo foi aprovado pelo Comitê de Ética em Pesquisa do Centro Universitário Univates, com número do parecer 198.599 e somente participaram do estudo os indivíduos que assinaram o termo de consentimento livre e esclarecido (TCLE), sendo excluídos os idosos que preenchessem o questionário de forma incorreta ou que desistissem de algum dos procedimentos do estudo.

A avaliação antropométrica foi composta pela aferição da circunferência da cintura (CC) e pelo controle do peso e da estatura para cálculo do índice de massa corporal (IMC). Para a aferição do peso dos indivíduos, utilizou-se uma balança portátil eletrônica, com capacidade máxima de $150 \mathrm{~kg}$ e precisão de 100 gramas. No momento da mensuração, o idoso foi orientado a vestir o mínimo de roupas, a ficar descalço e foi posicionado no centro da balança, de forma a distribuir o peso do corpo entre os pés (MASTROENI et al., 2010).

A aferição da estatura foi realizada com o auxílio de estadiômetro portátil, com capacidade de medição de $115 \mathrm{~cm}$ a $210 \mathrm{~cm}$. Os indivíduos permaneceram descalços, de costas para o estadiômetro, com os pés unidos, com os braços estendidos ao longo do corpo e em posição ereta, com a cabeça erguida, olhando para um ponto fixo na altura dos olhos (BUSNELLO, 2007). Com os dados de peso e estatura, foi calculado o IMC e classificado conforme os parâmetros de Lipschitz (1994): desnutridos com IMC $<22 \mathrm{~kg} / \mathrm{m}^{2}$, eutróficos com IMC entre 22 e $27 \mathrm{~kg} / \mathrm{m}^{2}$ e obesos/excesso de peso com IMC $>27 \mathrm{~kg} / \mathrm{m}^{2}$.
Avaliou-se, também, a circunferência da cintura (CC), que é uma medida utilizada para a avaliação da adiposidade abdominal e risco para o desenvolvimento de doenças cardiovasculares e distúrbios metabólicos (MORETTO et al., 2012). A CC foi classificada conforme os pontos de corte propostos pela World Health Organization (1998), que preconiza que circunferência abdominal $\geq 94 \mathrm{~cm}$ para homens e $\geq 80 \mathrm{~cm}$ para mulheres significa risco aumentado de complicações metabólicas associadas à obesidade, e circunferência abdominal $\geq 102 \mathrm{~cm}$ para homens e $\geq 88 \mathrm{~cm}$ para mulheres significa risco muito aumentado para complicações.

Os idosos responderam, ainda, a um questionário estruturado com questões referentes ao estilo de vida, tais como idade, estado civil, local de moradia, tabagismo, tempo de tabagismo, etilismo, prática de atividade física, além do diagnóstico de DCNT que foi autorreferido pelos participantes.

Para a análise estatística, as variáveis quantitativas foram descritas por média e desvio padrão ou mediana e amplitude interquartílica. As variáveis categóricas foram descritas por frequências absolutas e relativas. Para comparação das médias entre os grupos, o teste t-student foi aplicado. Em caso de assimetria, o teste de Mann-Whitney foi utilizado. Para comparar as proporções, os testes Qui-quadrado de Pearson ou exato de Fisher foram aplicados. O nível de significância adotado foi de $5 \%$ $(\mathrm{p} \leq 0,05)$ e as análises foram realizadas no programa Statistical Package for the Social Sciences (SPSS), na versão 18.0. 
Resultados

A população do estudo foi constituída por 112 indivíduos, com idade média de $66,1 \pm 4,1$ anos. Em relação à caracterização dos idosos, a maioria era do gênero feminino $(85,7 \%, \mathrm{n}=96)$, casada $(75,0 \%$, $\mathrm{n}=84)$ e residente da área urbana $(65,2 \%$, $\mathrm{n}=73$ ), conforme pode ser observado na Tabela 1. Os indivíduos do gênero masculino tinham maior idade $(\mathrm{p}=0,001)$, quando comparados às mulheres.
Em relação aos hábitos de vida, 5,4\% $(\mathrm{n}=6)$ dos idosos relataram tabagismo, sendo que entre os idosos do gênero masculino a prevalência de fumantes e o tempo de tabagismo foram estatisticamente maiores $(p=0,037 ; p=0,033)$, em comparação ao gênero feminino. Sobre o consumo de bebidas alcoólicas, os homens também apresentaram maior consumo $(\mathrm{p}=0,004)$.

Tabela 1: Características demográficas e estilo de vida de idosos do município de Roca Sales - RS (2013)

\begin{tabular}{|c|c|c|c|c|}
\hline Variáveis & $\begin{array}{l}\text { Amostra total } \\
\quad(n=112)\end{array}$ & $\begin{array}{c}\text { Mulheres } \\
(n=96)\end{array}$ & $\begin{array}{l}\text { Homens } \\
(n=16)\end{array}$ & $\mathrm{p}$ \\
\hline Idade (anos) - média \pm DP & $66,1 \pm 4,1$ & $65,6 \pm 3,9$ & $69,1 \pm 4,0$ & $0,001^{*}$ \\
\hline Estado civil - n(\%) & & & & $0,738^{* *}$ \\
\hline Solteiro & $2(1,8)$ & $2(2,1)$ & $0(0,0)$ & \\
\hline Casado & $84(75,0)$ & $72(75,0)$ & $12(75,0)$ & \\
\hline Divorciado & $14(12,5)$ & $11(11,5)$ & $3(18,8)$ & \\
\hline Viúvo & $12(10,7)$ & $11(11,5)$ & $1(6,3)$ & \\
\hline Local de moradia - $\mathrm{n}(\%)$ & & & & $1,000^{\star *}$ \\
\hline Área urbana & $73(65,2)$ & $63(65,6)$ & $10(62,5)$ & \\
\hline Área rural & $39(34,8)$ & $33(34,4)$ & $6(37,5)$ & \\
\hline Tabagista - n(\%) & & & & $0,037^{* * *}$ \\
\hline Sim & $6(5,4)$ & $3(3,1)$ & $3(18,8)$ & \\
\hline Não & $106(94,6)$ & $93(96,9)$ & $13(81,3)$ & \\
\hline $\begin{array}{l}\text { Tempo de tabagismo (anos) - } \\
\text { md (P25 - P75) }\end{array}$ & $25(20-35)$ & $20(17-30)$ & $35(23-48)$ & $0,033 \#$ \\
\hline Etilismo - n(\%) & & & & $0,004^{\star *}$ \\
\hline Raramente & $24(21,4)$ & $23(24,0)$ & $1(6,3)$ & \\
\hline Eventualmente & $24(21,4)$ & $16(16,7)$ & $8(50,0)$ & \\
\hline Frequentemente & $10(8,9)$ & $7(7,3)$ & $3(18,8)$ & \\
\hline Nunca & $54(48,2)$ & $50(52,1)$ & $4(25,0)$ & \\
\hline Prática de atividade física $-\mathrm{n}(\%)$ & & & & $0,163^{\star \star}$ \\
\hline Sim & $70(62,5)$ & $63(65,6)$ & $7(43,8)$ & \\
\hline Não & $42(37,5)$ & $33(34,4)$ & $9(56,3)$ & \\
\hline
\end{tabular}

Nota: *Teste t-student, **Teste qui-quadrado, ***Teste exato de Fisher, \#Teste Mann-Whitney, DP = desvio padrão, md = mediana. 
$\mathrm{Na}$ avaliação nutricional, descrita na Tabela 2, observou-se que os indivíduos do gênero masculino tiveram maior média de peso corpóreo e altura $(p=0,011$, $\mathrm{p}<0,001$ ), quando comparado ao feminino. Conforme o IMC, constatou-se que $57,1 \%(\mathrm{n}=64)$ dos idosos apresentavam excesso de peso. Em relação à CC, a média foi maior entre o gênero masculino $(\mathrm{p}<0,001)$. A maioria dos participantes $(82,1 \%, \mathrm{n}=92)$ apresentava risco muito aumentado para complicações metabólicas, porém, não houve diferença estatisticamente significativa entre os gêneros.

Tabela 2: Avaliação do estado nutricional de idosos do município de Roca Sales - RS (2013)

\begin{tabular}{|c|c|c|c|c|}
\hline Variáveis & $\begin{array}{l}\text { Amostra total } \\
\quad(n=112)\end{array}$ & $\begin{array}{l}\text { Mulheres } \\
(n=96)\end{array}$ & $\begin{array}{l}\text { Homens } \\
(n=16)\end{array}$ & $\mathrm{p}$ \\
\hline Peso $(\mathrm{kg})-$ média $\pm \mathrm{DP}$ & $69,7 \pm 13,1$ & $68,4 \pm 12,9$ & $77,3 \pm 11,7$ & $0,011^{*}$ \\
\hline Altura $(m)-$ média $\pm D P$ & $1,60 \pm 0,07$ & $1,58 \pm 0,06$ & $1,69 \pm 0,04$ & $<0,001^{*}$ \\
\hline IMC $\left(\mathrm{kg} / \mathrm{m}^{2}\right)-$ média $\pm \mathrm{DP}$ & $27,0 \pm 4,9$ & $27,1 \pm 5,0$ & $26,6 \pm 4,0$ & $0,730^{*}$ \\
\hline Classificação do IMC - n(\%) & & & & $0,901^{\star *}$ \\
\hline Desnutrição & $17(15,2)$ & $14(14,6)$ & $3(18,8)$ & \\
\hline Eutrofia & $31(27,7)$ & $27(28,1)$ & $4(25,0)$ & \\
\hline Excesso de peso & $64(57,1)$ & $55(57,3)$ & $9(56,3)$ & \\
\hline $\mathrm{CC}(\mathrm{cm})-$ média $\pm \mathrm{DP}$ & $98,6 \pm 11,2$ & $96,6 \pm 10,4$ & $110 \pm 9,0$ & $<0,001^{*}$ \\
\hline Classificação da CC - n(\%) & & & & $0,370^{\star \star}$ \\
\hline Normal & $7(6,3)$ & $7(7,3)$ & $0(0,0)$ & \\
\hline Aumentado & $13(11,6)$ & $10(10,4)$ & $3(18,8)$ & \\
\hline Muito aumentado & $92(82,1)$ & $79(82,3)$ & $13(81,3)$ & \\
\hline
\end{tabular}

Nota: *Teste t-student, **Teste qui-quadrado, DP = desvio padrão.

Quanto ao diagnóstico de DCNT, verificou-se que 49,1\% ( $n=55)$ eram diagnosticados com HAS, 39,3\% $(\mathrm{n}=44)$ com dislipidemia, $35,7 \%(\mathrm{n}=40)$ com osteoporose e $23,2 \%$ ( $n=26)$ com DM. Dos idosos avaliados, $83,0 \%(n=92)$ apresentaram pelo menos uma DCNT e o número médio

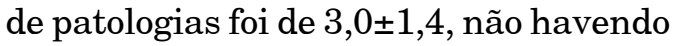
diferença estatisticamente significativa entre os gêneros $(p=0,465)$. Na Figura 1 , pode-se observar a associação entre diagnóstico de DCTN e gênero. 
Figura 1: Relação entre a prevalência de DCTN e gênero em idosos do município de Roca Sales $-\operatorname{RS}(2013)$

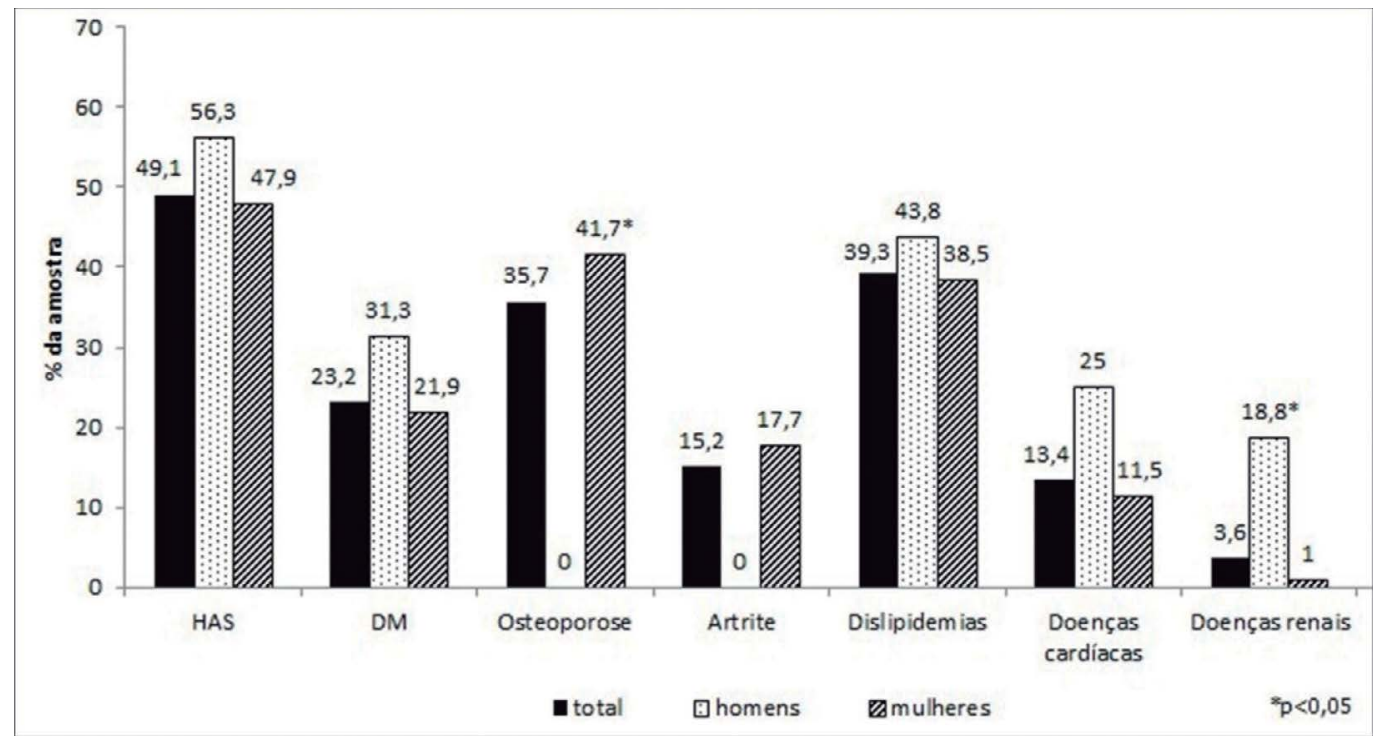

Na Tabela 3, encontra-se a associa- peso $(\mathrm{p}<0,001)$, quando comparados com ção entre a classificação do estado nutri- os eutróficos e desnutridos. Não houve cional pelo IMC e o diagnóstico de DCNT. associação estatisticamente significativa Pode-se verificar maior prevalência de entre desnutrição e eutrofia com a preHAS e DM em idosos com excesso de valência de DCNT.

Tabela 3: Relação entre a classificação nutricional pelo IMC e a prevalência de DCNT em idosos do município de Roca Sales - RS (2013)

\begin{tabular}{l|r|r|r|r}
\hline \multicolumn{1}{c|}{ Variáveis } & $\begin{array}{c}\text { Desnutrição } \\
(\mathrm{n}=17)\end{array}$ & $\begin{array}{c}\text { Eutrofia } \\
(\mathrm{n}=31)\end{array}$ & $\begin{array}{r}\text { Excesso de peso } \\
(\mathrm{n}=64)\end{array}$ & $\mathrm{p}^{*}$ \\
\hline Hipertensão arterial & $1(5,9)$ & $10(32,3)$ & $44(68,8)$ & $<0,001$ \\
Diabetes Mellitus & $1(5,9)$ & $0(0,0)$ & $25(39,1)$ & $<0,001$ \\
Osteoporose & $9(52,9)$ & $10(32,3)$ & $21(32,8)$ & 0,273 \\
Artrite & $2(11,8)$ & $3(9,7)$ & $12(18,8)$ & 0,468 \\
Dislipidemias & $5(29,4)$ & $9(29,0)$ & $30(46,9)$ & 0,165 \\
Patologias cardíacas & $2(11,8)$ & $1(3,2)$ & $12(18,8)$ & 0,112 \\
Patologias renais & $0(0,0)$ & $2(6,5)$ & $2(3,1)$ & 0,493 \\
\hline
\end{tabular}

Nota: * Teste qui-quadrado de Pearson. 
$\mathrm{Na}$ associação entre a $\mathrm{CC}$ e o diagnóstico de DCNT, pode-se observar relação significativa entre o diagnóstico de HAS e CC muito elevada ( $\mathrm{p}=0,002)$. Em relação às demais patologias, não houve diferença estatisticamente significativa, conforme apresentado na Tabela 4.

Tabela 4: Relação entre a classificação da CC e a prevalência de DCNT em idosos do município de Roca Sales - RS (2013)

\begin{tabular}{l|c|c|r|c}
\hline \multicolumn{1}{c|}{ Variáveis } & $\begin{array}{c}\text { Normal } \\
(\mathrm{n}=7)\end{array}$ & $\begin{array}{c}\text { Aumentada } \\
(\mathrm{n}=13)\end{array}$ & $\begin{array}{c}\text { Muito aumentada } \\
(\mathrm{n}=92)\end{array}$ & $\mathrm{p}^{*}$ \\
\hline HAS & $0(0,0)$ & $3(23,1)$ & $52(56,5)$ & 0,002 \\
DM & $0(0,0)$ & $2(15,4)$ & $24(26,1)$ & 0,224 \\
Osteoporose & $6(85,7)$ & $2(15,4)$ & $32(34,8)$ & 0,007 \\
Artrite & $2(28,6)$ & $2(15,4)$ & $13(14,1)$ & 0,590 \\
Dislipidemias & $3(42,9)$ & $4(30,8)$ & $37(40,2)$ & 0,792 \\
Patologias cardíacas & $2(28,6)$ & $1(7,7)$ & $12(13,0)$ & 0,414 \\
Patologias renais & $0(0,0)$ & $1(7,7)$ & $3(3,3)$ & 0,629 \\
\hline
\end{tabular}

Nota: * Teste qui-quadrado de Pearson.

\section{Discussão}

O presente estudo teve amostra constituída por idosos, em sua maioria, do gênero feminino e casados. Porém, o gênero masculino apresentou maiores médias de peso, estatura e CC. As DCNT mais recorrentes foram HAS, osteoporose e DM, e o número médio de patologias por idoso foi de três. Houve uma maior prevalência de HAS e DM em idosos com excesso de peso, em relação aos indivíduos eutróficos ou desnutridos.

A predominância do gênero feminino na amostra avaliada foi semelhante às encontradas nos estudos de Figueiredo et al. (2012), que observaram $72,3 \%$ de mulheres, de Moretto et al. (2012), em que $67,3 \%$ eram mulheres; e de Bueno et al. (2008), cujo estudo apresentou $90,2 \%$ dos idosos do gênero feminino. $\mathrm{O}$ estado civil da maioria dos idosos avaliados era de casado, assim como no estudo realizado por Neman e Silva (2011), no qual verificaram que $77,5 \%$ dos idosos eram casados.

Quanto à variável idade, os indivíduos do gênero masculino apresentaram idade superior em comparação ao feminino, assim como nos estudos de Barbosa et al. (2007), em que a idade média dos homens foi de 73,7 anos e das mulheres 72,8 anos; e de Piccoli et al. (2012), nos quais os homens apresentaram idade média de 69,5 anos e as mulheres 67,5 anos. Esse resultado corrobora com 0 aumento da longevidade dos idosos brasileiros estimada pelo Instituto Brasileiro de Geografia e Estatística (BRASIL, 2010).

No presente estudo, somente $5,4 \%$ da amostra total relatou ser tabagista, concordando com estudo de Cavalcanti et al. (2009), em que apenas $4,3 \%$ dos idosos 
informaram ter o hábito de fumar. Entre os homens, houve maior prevalência de fumantes, assim como nos estudos de Scherer e Vieira (2010), nos quais 11,5\% dos entrevistados eram tabagistas, e de Farias e Santos (2012), em que 8,0\% dos idosos eram fumantes.

Em relação ao consumo de bebidas alcoólicas, o presente estudo apontou maior consumo por parte dos indivíduos do gênero masculino, concordando com Farias e Santos (2012), que verificaram o consumo de bebidas alcoólicas em 92,3\% dos homens; e com Castillo et al. (2008), cujo estudo mostrou que $62,2 \%$ dos homens e $31,3 \%$ das mulheres reportaram o consumo de bebidas alcoólicas.

Assim como no presente estudo, Menezes e Marucci (2005), que avaliaram 305 idosos, e Machado, Coelho e Coelho (2010), que aferiram 395 idosos, também encontraram médias de peso e estatura mais elevadas no gênero masculino. Nesse estudo, a maioria dos idosos foi classificada com excesso de peso pelo IMC e a minoria com desnutrição ou baixo peso, concordando com os estudos de Bassler e Lei (2008), nos quais os autores verificaram $57,4 \%$ de sobrepeso, $33,0 \%$ de eutrofia e $9,6 \%$ de baixo peso. Concordam também com os achados de Bueno et al. (2008), com maior prevalência de $52,4 \%$ de sobrepeso, $28,1 \%$ de eutrofia e $19,5 \%$ de baixo peso; e de Nascimento et al. (2011), com $45,5 \%$ de excesso de peso e $13,6 \%$ de baixo peso.

A maioria dos participantes encontrava-se com risco muito aumentado de complicações metabólicas associadas à obesidade pela CC. Resultado semelhante também foi observado por Fiore et al.
(2006), que avaliaram 73 idosos frequentadores de unidades básicas de saúde, sendo que $67,1 \%$ da amostra apresentava CC muito aumentada. Scherer e Vieira (2010), em estudo realizado com 199 idosos em Marques de Souza-RS, e Rocha et al. (2013), que estudaram 321 idosos de Campina Grande-PB, também encontraram médias da CC maiores em indivíduos do gênero masculino, quando comparado ao feminino. Outros autores também encontraram CC aumentada em idosos, o que caracteriza um importante fator de risco para o desenvolvimento de doenças cardiovasculares, e DCNT (CARVALHO et al., 2011; KÜMPEL et al., 2011).

Em relação às DCNT, as mais recorrentes foram HAS, osteoporose e DM. Resultados semelhantes foram observados no estudo de Cavalcanti et al. (2009), em que a HAS foi a patologia mais prevalente, com $56,4 \%$, e DM, com $20,5 \%$. Entretanto, nesse estudo, a osteoporose apareceu somente como quinta patologia mais prevalente, com $12,0 \%$. No estudo de Bassler e Lei (2008), as três patologias mais citadas pelos idosos foram HAS $(59,8 \%)$, dislipidemias $(20,6 \%)$ e DM $(15,5 \%)$. A osteoporose foi pouco mencionada, somente $2,1 \%$ dos idosos afirmaram ter diagnóstico. Conforme o estudo de Alves et al. (2007), HAS foi a patologia crônica mais frequente $(53,4 \%)$.

No estudo de Cavalcanti et al. (2009), $82,1 \%$ dos idosos afirmaram possuir diagnóstico de alguma patologia. Pinelli et al. (2005) também observaram que $66,0 \%$ dos participantes tinham ao menos uma DCNT, também concordando com os dados observados. 
Os achados de associação entre IMC elevado e HAS/DM também foram verificados por outros autores, tais como Munaretti et al. (2011), em estudo transversal com 1894 idosos. Além disso, Portela, Pontes e Pretto (2007), em pesquisa realizada com 54 idosos de Picos-PI, também observaram associação entre IMC elevado e HAS. Francisco et al. (2010), ao estudarem 2151 idosos, e Aurichio, Rebelatto e Castro (2010), em estudo realizado com 399 idosos, também encontraram associação entre excesso de peso e diagnóstico de DM.

Neto et al. (2008) analisaram a CC de 131 idosos hipertensos e verificaram que $95,9 \%$ das mulheres e $52,9 \%$ dos homens apresentaram risco aumentado para complicações metabólicas, assim como Freitas et al. (2012), que observaram, numa amostra de 124 hipertensos, que $89,0 \%$ das mulheres e $64,3 \%$ dos homens apresentaram risco aumentado para o desenvolvimento de doenças cardiovasculares e metabólicas. Tais dados mostram-se em conformidade com os achados do presente estudo.

Como limitações do estudo, destaca-se o número amostral decorrente da baixa adesão dos idosos aos grupos de terceira idade do município. Entretanto, esse é um município pequeno e com reduzido número de habitantes, onde praticamente metade da população reside na área rural. Ademais, utilizou-se o IMC para avaliação nutricional por ser prático, rápido e de baixo custo. Porém, esse parâmetro foi avaliado em conjunto com a circunferência da cintura, visando minimizar o uso isolado do IMC.

\section{Conclusões}

Diante dos resultados obtidos neste estudo, pode-se observar alta prevalência de sobrepeso, risco muito elevado para complicações metabólicas associadas à obesidade e ao diagnóstico médio de três DCNT. Dentre as DCNT, as mais prevalentes foram HAS, osteoporose e DM. Houve associação entre excesso de peso pelo IMC e diagnóstico de HAS e $\mathrm{DM}$, além de associação entre CC muito elevada e o diagnóstico de HAS. Esses resultados sugerem que os idosos avaliados encontravam-se em condições nutricionais inadequadas. Conclui-se que é de extrema importância a criação de medidas preventivas e de intervenção, visando ao controle da obesidade e das DCNT.

\section{Association between nutritional status and prevalence of non-communicable chronic diseases in the elderly residents in the city of Roca Sales/RS}

\section{Abstract}

The study aims evaluate the nutritional status and associate it with the prevalence of chronic non-communicable diseases (NCDs) in the elderly. It is a cross-sectional study participated in 112 individuals, aged over 60 years, from both genders, participants in third age groups in the municipality of Roca Sales-RS. To evaluate nutritional status were measured waist circumference (WC), weight and height, was calculated the body mass index (BMI). It was applied a questionnaire for obtaining information about lifestyle and the diagnosis of NCDs was self-reported by participants. Observed that $85.7 \%(n=96)$ of participants were female and $75.0 \%(n=84)$ were married. According BMI was observed

RBCEH, Passo Fundo, v. II, n. 2, p. I66-177, maio/ago. 2014 
that $57.1 \%(n=64)$ were overweight, and men with higher mean BMI $(p=0.011)$. In the evaluation of the $\mathrm{WC}$, the men also had a higher average than women $(p<0.001)$. The majority of participants $(82.1 \%, \mathrm{n}=92)$ had very increased risk for metabolic disorders. NCDs diagnosed have been: hypertension, osteoporosis and diabetes mellitus. Elderly subjects, $83,0 \%(n=92)$ had at least one and three pathology is the average number of pathologies. From the results of obtained showed overweight, very increased risk for metabolic disorders and diagnosis of pathology in most of the individuals.

Keywords: Nutritional status. Chronic disease. Health of the elderly.

\section{Referências}

ALVES, L. C. et al. A influência das doenças crônicas na capacidade funcional dos idosos do Município de São Paulo, Brasil. Cadernos de Saúde Pública, Rio de Janeiro, v. 23, n. 8, p. 1924-1930, ago. 2007.

AURICHIO, T. R.; REBELATTO, J. R.; CASTRO, A. P. Obesidade em idosos do Município de São Carlos, SP e sua associação com diabetes melito e dor articular. Fisioterapia e Pesquisa, São Paulo, v. 17, n. 2, p. 114-117, abr./jun. 2010.

BARBOSA, A. R. et al. Estado nutricional e desempenho motor de idosos de São Paulo. Revista da Associação Médica Brasileira, São Paulo, v. 53, n. 1, p. 75-79, jul./set. 2007.

BASSLER, T. C.; LEI, D. L. M. Diagnóstico e monitoramento da situação nutricional da população idosa em município da região metropolitana de Curitiba. Revista de Nutrição, Campinas, v. 21, n. 3, p. 311-321, maio/ jun. 2008.

BUENO, J. M. et al. Avaliação nutricional e prevalência de doenças crônicas não transmissíveis em idosos pertencentes a um programa assistencial. Ciência e Saúde Coletiva, Rio de Janeiro, v. 13, n. 4, p. 1237-1246, jul./ ago. 2008.
BUSNELLO, F. M. Aspectos nutricionais no processo do envelhecimento. São Paulo: Atheneu, 2007.

CARVALHO, C. A. et al. Nível de atividade física de servidores idosos em período de pré-aposentadoria da Universidade Federal do Maranhão - UFMA. Revista de Pesquisa em Saúde, São Luís, v. 12, n. 2, p. 32-37, maio/ago. 2011.

CASTILLO, B. A. A. et al. Situações estressantes de vida, uso e abuso de álcool e drogas em idosos de Monterrey, México. Revista Latino-Americana de Enfermagem, Ribeirão Preto, v. 16, n. 1, p. 509-515, jul./ago. 2008.

CAVALCANTI, C. L. et al. Prevalência de doenças crônicas e estado nutricional em um grupo de idosos brasileiros. Revista de Salud Pública, v. 11, n. 6, p. 865-877, dez. 2009.

FARIAS, R. G.; SANTOS, S. M. A. Influência dos determinantes do envelhecimento ativo entre idosos mais idosos. Texto Contexto Enfermagem, Florianópolis, v. 21, n. 1, p. 167-176, jan./mar. 2012.

FIGUEIREDO, V. R. et al. Characteristics of the population of an excellence center in healthcare for the elderly. Journal of Nursing UFPE On Line, Recife, v. 6, n. 11, p. 27442752, Dec. 2012.

FIORE, E. G. et al. Perfil nutricional de idosos frequentadores de unidade básica de saúde. Revista de Ciências Médicas, Campinas, v. 15, n. 5 p. 369-377, set./out. 2006.

FLORIANO, L. A. et al. Cuidado realizado pelo cuidador familiar ao idoso dependente, em domicílio, no contexto da Estratégia de Saúde da Família. Texto Contexto Enfermagem, Florianópolis, v. 21, n. 3, p. 543-548, jul./set. 2012.

FRANCISCO, P. M. S. B. et al. Diabetes auto-referido em idosos: prevalência, fatores associados e práticas de controle. Cadernos de Saúde Pública, São Paulo, v. 26, n. 1, p. 175-184, 2010. 
FREITAS, L. C. et al. Perfil dos hipertensos da Unidade de Saúde da Família Cidade Nova 8, município de Ananindeua-PA. Revista Brasileira de Medicina de Família e Comunidade, Florianópolis, v. 7, n. 22, p. 13-19, jan./mar. 2012.

INSTITUTO BRASILEIRO DE GEOGRAFIA E ESTATÍSTICA - IBGE. Censo Demográfico 2010. Rio de Janeiro, 2014. Disponível em: $<$ http://www.censo2010.ibge.gov.br>. Acesso em: 3 set. 2014 .

KÜMPEL, D. A. et al. Obesidade em idosos acompanhados pela Estratégia de Saúde da Família. Texto Contexto Enfermagem, Florianópolis, v. 20, n. 3, p. 471-477, jul./set. 2011.

LIMA, M. G. et al. Impact of chronic disease on quality of life among the elderly in the state of São Paulo, Brazil: a population-based study. Revista Panamericana de Salud Publica, Washington DC, v. 25, n. 4, p. 314-321, Apr. 2009.

LIPSCHITZ, D. A. Screening for nutritional status in the elderly. Primary Care, Philadelphia, v. 21, n. 1, p. 55-67, 1994.

MACHADO, R. S. P.; COELHO, M. A. S. C.; COELHO, K. S. C. Percentual de gordura corporal em idosos: comparação entre os métodos de estimativa pela área adiposa do braço, pela dobra cutânea triciptal e por bioimpedância tetrapolar. Revista Brasileira de Geriatria e Gerontologia, Rio de Janeiro, v. 13, n. 1, p. 17-28, 2010.

MASTROENI, M. F. et al. Antropometria de idosos residentes no município de Joinville-SC, Brasil. Revista Brasileira de Geriatria e Gerontologia, Rio de Janeiro, v. 13, n. 1, p. 29-40, 2010.

MENEZES, T. N.; MARUCCI, M. F. N. Antropometria de idosos residentes em instituições geriátricas, Fortaleza, CE. Revista de Saúde Pública, São Paulo, v. 39, n. 2, p. 169-175, 2005.

MONTENEGRO NETO, A. N. et al. Archivos Latinoamericanos de Nutricion, Caracas, v. 58, n. 4 , p. 350-356, dez. 2008.
MORETTO, M. C. et al. Relação entre estado nutricional e fragilidade em idosos brasileiros. Revista da Sociedade Brasileira de Clínica Médica, São Paulo, v. 10, n. 4, p. 267-71, jul./ago. 2012.

MUNARETTI, D. B. et al. Hipertensão arterial referida e indicadores antropométricos de gordura em idosos. Revista da Associação Médica Brasileira, São Paulo, v. 57, n. 1, p. 25-30, jan./fev. 2011.

NASCIMENTO, C. M. et al. Estado nutricional e condições de saúde da população idosa brasileira: revisão da literatura. Revista Medica de Minas Gerais, Belo Horizonte, v. 21, n. 2, p. 174-180, 2011.

NEMAN, F. A.; SILVA, N. H. O perfil de saúde dos indivíduos idosos residentes no município de Guarulhos. Revista Science in Health, São Paulo, v. 2, n. 2, p. 83-92, maio/ago. 2011.

OLIVEIRA, S. F. D. et al. Demanda referida e auxílio recebido por idosos com declínio cognitivo no município de São Paulo. Saúde e Sociedade, São Paulo, v. 16, n. 1, p. 81-89, jan./abr. 2007.

PEDRAZZI, E. C.; RODRIGUES, R. A. P.; SCHIAVETO, F. V. Morbidade referida e capacidade funcional de idosos. Ciência, Cuidado e Saúde, Maringá, v. 6, n. 4, p. 407-413, out./dez. 2007.

PICCOLI, J. C. J. et al. Coordenação global, equilíbrio, índice de massa corporal e nível de atividade física: um estudo correlacional em idosos de Ivoti, RS, Brasil. Revista Brasileira de Geriatria e Gerontologia, Rio de Janeiro, v. 15, n. 2, p. 209-222, 2012.

PINELLI, L. A. P. et al. Prevalência de doenças crônicas em pacientes geriátricos. Revista Odonto Ciência - PUCRS, Porto Alegre, v. 20, n. 47, p. 69-74, jan./mar. 2005.

PORTELA, B. O.; PONTES, L. M.; PRETTO, A. C. Prevalência de obesidade em normotensos e hipertensos assistidos pela Unidade de Saúde da Família (USF) do bairro parque de exposição, Picos - Piauí. Coleção Pesquisa em Educação Física, São Paulo, v. 6, n. 2, p. 449-456, jul. 2007. 
ROCHA, F. L. et al. Correlação entre indicadores de obesidade abdominal e lipídeos séricos em idosos. Revista da Associação Médica Brasileira, São Paulo, v. 59, n. 1, p. 48-55, jan./fev. 2013.

SCHERER, F.; VIEIRA, J. L. C. Estado nutricional e sua associação com risco cardiovascular e síndrome metabólica em idosos. Revista de Nutrição, Campinas, v. 23, n. 3, p. 347-355, maio/jun. 2010.

VERAS, R. P. Estratégias para o enfrentamento das doenças crônicas: um modelo em que todos ganham. Revista Brasileira de Geriatria e Gerontologia, Rio de Janeiro, v. 12 , n. 4, p. 779-786, 2011.

WORLD HEALTH ORGANIZATION. Physical status: the use and interpretation of anthropometry. Geneva: World Health Organization, 1995.

Obesity: preventing and managing the global epidemic. Geneva: World Health Organization, 1998. 